\title{
Clathrin-independent endocytosis: from nonexisting to an extreme degree of complexity
}

\author{
Kirsten Sandvig • Maria Lyngaas Torgersen • \\ Hilde Andersen Raa $\cdot$ Bo van Deurs
}

Accepted: 24 December 2007 / Published online: 12 January 2008

(C) Springer-Verlag 2008

\begin{abstract}
Today it is generally accepted that there are several endocytic mechanisms, both the clathrin-dependent one and mechanisms which operate without clathrin and with different requirements when it comes to dynamin, small GTP-binding proteins of the Rho family and specific lipids. It should be noted that clathrin-independent endocytosis can occur even when the cholesterol level in the membrane has been reduced to so low levels that caveolae are gone and clathrin-coated membrane areas are flat. Although new investigators in the field take it for granted that there is a multitude of entry mechanisms, it has taken a long time for this to become accepted. However, more work needs to be done, because one can still ask the question: How many endocytic mechanisms does a cell have, what are their function, and how are they regulated? This article describes some of the history of endocytosis research and attempts to give an overview of the complexity of the mechanisms and their regulation.
\end{abstract}

Keywords Endocytosis - Rho proteins - Caveolae . Flotillin $\cdot$ Ricin $\cdot$ Shiga toxin $\cdot$ Cholera toxin

\footnotetext{
K. Sandvig $(\varangle) \cdot$ M. L. Torgersen $\cdot$ H. A. Raa

Centre for Cancer Biomedicine,

University of Oslo and Department of Biochemistry,

The Norwegian Radium Hospital,

Montebello 0310 Oslo, Norway

e-mail: ksandvig@ radium.uio.no

K. Sandvig $\cdot$ H. A. Raa

Department of Molecular Biosciences,

University of Oslo, 0316 Oslo, Norway

B. van Deurs

Department of Cellular and Molecular Medicine,

University of Copenhagen, The Panum Institute,

2200 Copenhagen N, Denmark
}

\section{Introduction}

The existence of different endocytic mechanisms is becoming of increasing interest, for instance in relation to growth and differentiation, cell adhesion, regulation of the activity of signalling receptors, drug delivery, and entry of pathogens. The most well-studied endocytic mechanism involves clathrin-coated pits and vesicles, and impressive amounts of details are now known about clathrin-dependent endocytosis (Schmid and McMahon 2007; Benmerah and Lamaze 2007; Ungewickell and Hinrichsen 2007). Initially, the idea was that the formation of the clathrin basket was the driving force during membrane internalization, and quantitative studies suggested that the pathway could account for everything endocytosed (Doxsey et al. 1987). With basic ideas such as "biology is simple" and questions like, "how can a vesicle form without clathrin?", it was difficult and it took time to get acceptance for the existence of alternative endocytic pathways (Montesano et al. 1982; Sandvig et al. 1985, 1987; Moya et al. 1985; Payne et al. 1988; Sandvig and van Deurs 1991, 1994; Damke et al. 1995). Clathrin-dependent endocytosis can be inhibited both by removing clathrin from the plasma membrane by a treatment involving hypotonic shock and depletion of cytosolic potassium (Larkin et al. 1983), and by "freezing" the clathrin coat at the surface by acidification of the cytosol (Sandvig et al. 1987, 1988). The finding that endocytosis of for instance the protein toxin ricin continued under both conditions, suggesting that clathrin-independent endocytosis was responsible for this uptake, was apparently difficult to accept for a number of investigators during a 10 year period (1985-1995). New techniques in molecular biology have been crucial for the elucidation of endocytic processes, and when in 1995 it was published that the dynamin-mutant K44A inhibited clathrin-mediated endocytosis and that the cells still 
endocytosed (Damke et al. 1995), the complexity of endocytosis started to become apparent to the community in general. Not only did clathrin-independent endocytosis exist, it could also be regulated (Damke et al. 1995). We now know that a number of signalling pathways can regulate clathrin-independent endocytosis (for review, see Sandvig and van Deurs 2005; Marsh and Helenius 2006; Mayor and Pagano 2007). Despite accumulating evidence for several endocytic mechanisms, partly obtained by studies of toxins and viruses (Marsh and Helenius 2006; Sandvig and van Deurs 2002), many text-books in cell biology still operate with only two endocytic mechanisms, uptake from clathrin-coated pits and caveolae. The aim of this brief review is to outline the extreme complexity of clathrin-independent endocytosis as it appears today.

\section{Clathrin-independent endocytosis: expansion of the field}

The number of clathrin-independent endocytic pathways revealed has been increasing with time. Mayor and Pagano (Mayor and Pagano 2007) recently subdivided the different mechanisms into four types: RhoA-regulated, cdc42-regulated, and Arf6-dependent mechanisms, and caveolar uptake. Of these, the RhoA-regulated mechanism and caveolar endocytosis also require the GTP-binding protein dynamin (Fig. 1a). However, there are already reports of mechanisms that may not necessarily fit into any of these four categories. For instance, clathrin-independent internalization of proteoglycans seems to depend not only on dynamin, but also on flotillin (Payne et al. 2007), and internalization of Herpes Simplex Virus Protein VP22 is independent of clathrin, but dependent on dynamin and Arf6 (Nishi and Saigo 2007). Also, studies of virus and toxin uptake indicate that there could be even more mechanisms (Marsh and Helenius 2006; Sandvig 2001; Sandvig and van Deurs 2005). Since cells may compensate for loss of one endocytic pathway by upregulating another (Damke et al. 1995), one can of course also question to which extent interfering with the endocytic machinery of physiologically relevant pathways in different ways, might create new pathways or cause upregulation of those normally playing only a minor role. Today, more specific methods such as RNA interference (RNAi) are used to interfere with a given process. However, also RNAi-mediated removal of a given molecule can induce upregulation of related molecules (Utskarpen et al. 2006). Moreover, upon removal of a membrane-associated molecule one may ask: To which extent do we actually know that the endocytic process is blocked? Could the rest of the machinery allow uptake to continue with different kinetics and be interpreted as a "new mechanism"? Similarly, an old objection to the notion that
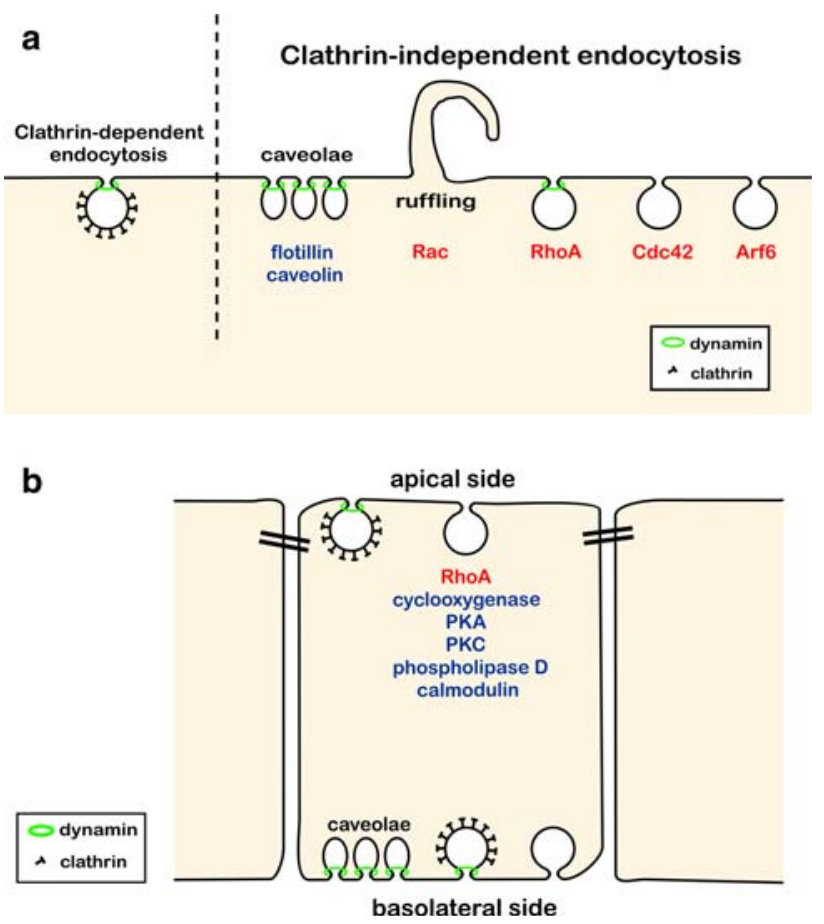

Fig. 1 An overview of a factors involved in different types of endocytosis, and $\mathbf{b}$ schematic drawing of polarized MDCK cells where some proteins/enzymes involved in regulation of apical clathrin-independent endocytosis have been listed. It should be noted that apical clathrinindependent endocytosis may consist of more than one mechanism and that polarized MDCK cells have caveolae only at the basolateral surface. For references, see the text

removal of clathrin by potassium-depletion (Larkin et al. 1983; Moya et al. 1985; Madshus et al. 1987; Sandvig et al. 1985) revealed endocytosis normally operating in parallel to the clathrin-dependent pathway, was that the rest of the endocytic machinery originally belonging to the clathrindependent machinery was still operating. A similar critical attitude should be applied on today's interpretations. Importantly, both toxins and viruses can modify endocytic events, in some instances by inducing signalling, even though they may bind to glycolipid receptors (Marsh and Helenius 2006; Lauvrak et al. 2006; Römer et al. 2007).

Internalization of membrane components and ligands by endocytosis thus have different requirements when it comes to dependency of dynamin, caveolin, flotillin, and small GTPases, and in addition, cholesterol and other specific lipids play a role (see below). To which extent the different endocytic mechanisms vary depending on cell type, growth conditions and the extent of cell polarization is to a large extent not known, and has to be addressed in future studies. It should be stressed that most of our knowledge about clathrin-independent endocytic mechanisms derives from cells in culture.

The work of combining different results into clearly defined models is far from finished. In 2005 it was published 
that $\mathrm{CtBP} 3 / \mathrm{BARS}$ drives membrane fission in processes where dynamin is not involved (Bonazzi et al. 2005). Interfering with the action of this protein reduces fluid phase uptake, as previously reported for cdc42-dependent uptake (Mayor and Pagano 2007). However, whether cdc42 and CtBP3/BARS affect the same process does not seem to be known. The possibility exists that CtBP3/BARS might indirectly affect endocytosis (Mayor and Pagano 2007). Furthermore, when inhibiting endocytic uptake of a ligand, it can be difficult to measure whether it is movement of the ligand into the membrane invagination giving rise to the endocytic vesicle that is reduced or whether it is the formation of the vesicle that is the step affected. To classify the different mechanisms properly, more information is required (see Fig. 1a, b). It is important to note that polarized cells have a differential regulation of clathrin-independent endocytosis at the two poles, and regulators of apical clathrin-independent endocytosis have been indicated in Fig. 1b (separate chapter below). Interestingly, caveolae are only present on the basolateral side in the polarized epithelial cell line MDCK (Verkade et al. 2000; Vogel et al. 1998). In the following sections we will describe various aspects of the clathrin-independent mechanisms in more detail.

\section{Membrane lipids required for clathrin-independent endocytosis}

In addition to a large number of proteins, also membrane lipids are important for clathrin-independent endocytosis. Interfering with sphingolipid synthesis may affect different types of clathrin-independent endocytosis by mechanisms poorly understood (Cheng et al. 2006). Importantly, sphingomyelin seems to be required both for recruitment of RhoA and cdc42 to the membrane and for endocytosis involving these GTP-binding proteins (Cheng et al. 2006). Cdc42-dependent uptake is independent not only of RhoA, but also of Rac, clearly separating it from macropinocytosis which requires Rac activity (for review, see (Hanzal-Bayer and Hancock 2007; Chadda et al. 2007). Interestingly, sphingolipid synthesis, or rather the production of glycosphingolipids was found to be required for transport of caveolin to the plasma membrane and for caveolae formation (Cheng et al. 2006).

A change in the fatty acid composition of membrane lipids can also be expected to change the properties of the membrane and its interaction with cytosolic proteins of importance for endocytosis. It was recently found that incubation of cells with unsaturated fatty acids affect the uptake of the glycolipid-binding Shiga toxin which is internalized both by clathrin-dependent and clathrin-independent endocytosis (Spilsberg et al. 2007). A possible explanation for this finding is that there is a change in the amount and/or size of rafts or their ability to become associated with the endocytic machinery. Lipid rafts play a central role in membrane structure and function (Rajendran and Simons 2005), and it is therefore not surprising that the fatty acid composition may affect endocytosis. Raft-dependent uptake have recently been reviewed (Marsh and Helenius 2006; Lajoie and Nabi 2007; Hanzal-Bayer and Hancock 2007).

One can often see the generalized statement: clathrinindependent endocytosis is cholesterol-dependent (HanzalBayer and Hancock 2007). This is true for processes such as macropinocytosis (Grimmer et al. 2002), uptake from caveolae, Rho-dependent uptake of IL2 (a recent review has been written by Lajoie and Nabi 2007), and cdc42dependent endocytosis where it was reported that there is a cholesterol-dependent activation of cdc42 which in turn mediates actin polymerization required for endocytosis via this pathway (Chadda et al. 2007). However, a normal concentration of cholesterol does not seem to be required for uptake of all ligands taken in by clathrin-independent endocytosis (Rodal et al. 1999). When enough cholesterol has been extracted from the plasma membrane for the caveolae to disappear and the clathrin-coated pits to flatten, endocytosis of the plant toxin ricin still occurs (Rodal et al. 1999). Furthermore, when Cholera toxin uptake into HeLa cells is reduced by expression of the dynamin-mutant K44A or by induction of antisense to clathrin heavy chain in BHK cells, endocytosis of this toxin can be increased by extraction of cholesterol with methyl- $\beta$-cyclodextrin (Sandvig et al. 2004). Similarly, uptake of Shiga toxin is increased by treatment with methyl- $\beta$-cyclodextrin after block of clathrin-dependent uptake by induced expression of antisense to clathrin heavy chain in BHK cells (Fig. 2). One possible explanation is that these toxins are retained at the cell surface by being associated with rafts, and that release from these structures by treatment with methyl- $\beta$-cyclodextrin actually permits endocytosis of the toxins. Also, uptake of proteoglycan-binding ligands, reported to be clathrin- and caveolin-independent, but dynamin- and flotillin-dependent, were unaffected by filipin and nystatin, drugs known to disrupt lipid rafts (Payne et al. 2007). It should be noted that cholesterol-depletion can inhibit the formation of invaginated clathrin-coated pits by a so far unknown process (Rodal et al. 1999; Subtil et al. 1999), and cholesteroldependency is therefore not necessarily a tool to easily distinguish between endocytic processes.

\section{Role of actin in endocytosis}

Recruitment of actin to clathrin-coated pits and subsequent actin-driven formation and internalization of endocytic vesicles and the appearance of actin "comet tails" on such 


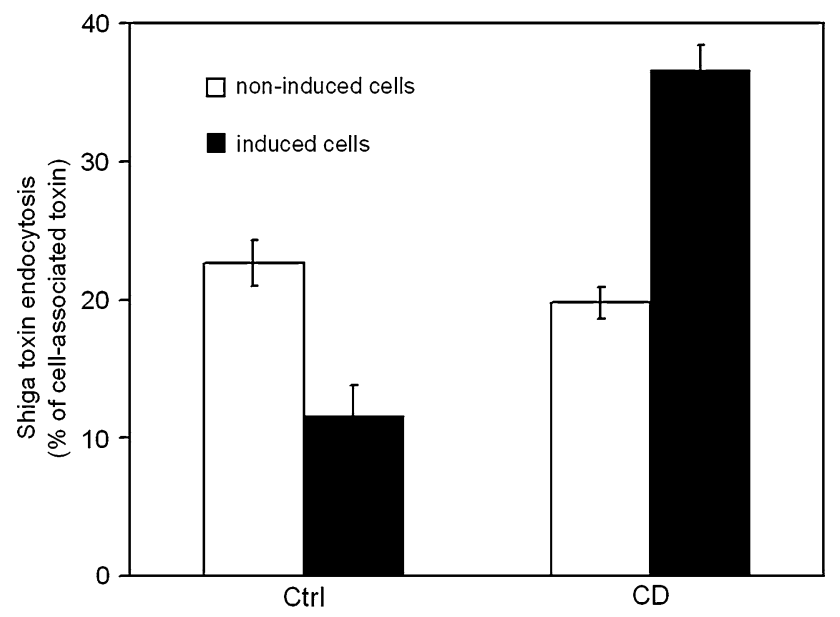

Fig. 2 Shiga toxin endocytosis is strongly increased upon methyl- $\beta$ cyclodextrin-treatment of BHK cells induced for expression of antisense to clathrin heavy chain. BHK cells with inducible expression of antisense to clathrin heavy chain was preincubated with methyl- $\beta$ cyclodextrin $(10 \mathrm{mM})$ for $30 \mathrm{~min}$. Then biotin- and TAG-labeled Shiga toxin $(25 \mathrm{ng} / \mathrm{ml})$ was added and the cells were incubated for $20 \mathrm{~min}$. Surface-bound and endocytosed Shiga toxin was quantified as previously described for Cholera toxin (Torgersen et al. 2001). The data are presented as internalized toxin as percent of total cell-associated toxin (mean $\pm \mathrm{SD}, n=4$ ). Endocytosis of transferrin was performed in parallel to verify the inhibition of clathrin-dependent endocytosis upon induction of antisense to clathrin heavy chain. Upon induction, transferrin uptake was reduced by $95 \%$

vesicles is well-established and appears to be a highly complex process involving several protein systems (McPherson 2002; Kaksonen et al. 2005, 2006; Smythe and Ayscough 2006). Interestingly, a crucial role of actin in clathrin-independent endocytosis is now becoming evident as well (Kaksonen et al. 2006; Yarar et al. 2007; Lanzetti 2007), and it seems likely that actin will turn out to be involved in all clathrin-independent endocytic mechanisms. However, in some cases actin might function to facilitate the process and not be strictly required. It has for instance been reported that there is a difference between cells growing in monolayers and cells in suspension when it comes to actin requirement for clathrin-dependent endocytosis (Fujimoto et al. 2000). One of the most studied examples of a clathrinindependent endocytic mechanism that is based on actin polymerization, is macropinocytosis (see below), a major fluid-phase uptake pathway.

\section{Macropinocytosis}

An increasing complexity of the formation of macropinosomes is becoming evident. Thus, it has long been known that GTP-bound Rac stimulates accumulation of actin filaments at the plasma membrane and that it is involved in plasma membrane ruffling induced by growth factors
(Ridley et al. 1992) (Fig. 1a). Rac in turn activates the Arp2/3 protein complex and WAVE, a process involved in ruffling (Gao et al. 2007). In addition to Rac, also Ras, Src and phosphoinositide (PI) 3-kinase promote macropinocytosis (Gao et al. 2007), and a role of N-WASP and the sorting nexin 9 (SNX9) in assembly and organization of actin during ruffle formation and macropinocytosis has recently been shown (Yarar et al. 2007). Also, histone deacetylase 6 (HDAC6) is associated with plasma membrane ruffles and formation of macropinosomes upon stimulation with growth factors, processes that were inhibited in HDAC6deficient cells. Interestingly, a substrate of HDAC6, the heat shock protein Hsp90, seemed to be involved in ruffling and macropinocytosis as well (Gao et al. 2007). Moreover, a clathrin-independent process reminiscent of macropinocytosis has recently been reported (Orth et al. 2006). Here, actin-dependent circular dorsal ruffles or dorsal membrane waves are formed upon growth factor stimulation, leading to significant receptor internalization. Like in ordinary macropinocytosis, PI 3-kinase is involved, but interestingly, also dynamin, which is a part of the actin-polymerizing Arp2/3-N-WASP-cortactin system and a large number of other proteins are required for this internalization mechanism (Orth et al. 2006; Orth and McNiven 2006; McNiven 2006).

\section{RhoA-, cdc42- and CTBP3/BARS-dependent uptake}

The RhoA-dependent uptake of IL-2 receptors, $\gamma c$-cytokine receptor and IgE receptor is a dynamin-dependent process (Mayor and Pagano 2007) (Fig. 1). Whether RhoA is required for the correct sorting of ligand or whether it is involved in the endocytic process as such is however being discussed (Mayor and Pagano 2007). Data from polarized cells showing that ricin uptake and fluid phase endocytosis at the apical side of polarized MDCK cells are also dependent on RhoA (Garred et al. 2001), suggest that it is the endocytic mechanism itself that is RhoA-dependent.

A cdc42-regulated process, which operates independently of dynamin, has been reported to be involved in uptake of glycosylphosphatidylinositol (GPI)-anchored proteins as well as being responsible for a major fraction of fluid-phase uptake in the cell (Kalia et al. 2006). GPIanchored proteins were found to be internalized into $\mathrm{CHO}$ cells by a cdc42-dependent, but Arf6-independent mechanism. The internalized membrane ended up in vesicles without EEA1, Rab4, or Rab5, but these vesicles were found to fuse with normal endosomal compartments via a Rab5/PI3-kinase dependent machinery. That a GPIanchored protein and its ligand can enter independently of caveolae and dynamin and reach an acidic compartment, was first shown in studies of a GPI-anchored diphtheria 
toxin receptor (Skretting et al. 1999). The finding that diphtheria toxin bound to this GPI-linked receptor intoxicates cells, demonstrates that the toxin has been transferred to an acidic compartment facilitating low $\mathrm{pH}$-induced conformational change of diphtheria toxin and translocation of the toxin to the cytosol.

The finding that the cdc42-dependent mechanism is responsible for most fluid uptake (Kalia et al. 2006) is surprising, considering all the other uptake mechanisms now reported, not least macropinocytosis. It was recently shown that cdc42 is associated with the recycling endosome and that also Par proteins play a role in the endocytic process (Balklava et al. 2007). To which extent there is an indirect effect on the uptake from the plasma membrane when mutants of cdc42 are studied, is not quite clear. Also, as mentioned earlier, since CtBP3/BARS is involved in dynamin-independent endocytosis (Bonazzi et al. 2005), and also endocytosis regulated by this protein can account for a considerable amount of fluid phase uptake, it should be elucidated to which extent cdc 42 and $\mathrm{CtBP} 3 / \mathrm{BARS}$ regulated processes are related.

\section{Arf6-dependent uptake}

ADP-ribosylation factor 6 (Arf6) has been identified as a key regulator of a clathrin- and dynamin-independent endocytic pathway mainly studied in HeLa and COS-7 cells, and responsible for uptake and recycling of a collection of integral plasma membrane proteins devoid of conventional endocytic signals (see below). The number of cargo proteins reported to be internalized by Arf6-regulated endocytosis in these cells is increasing and includes the plasma membrane proteins Major Histocompatibility Complex Class I (MHCI) and interleukin-2 receptor $\alpha$-subunit/Tac (Radhakrishna and Donaldson 1997), $\beta 1$-integrin (Brown et al. 2001), M2 muscarinic acetylcholine receptor (Delaney et al. 2002) and certain GPI-anchored proteins, such as CD59 (Naslavsky et al. 2004). Internalized cargo can recycle to the plasma membrane via the tubular Arf6-positive recycling compartment (Radhakrishna and Donaldson 1997) or, alternatively, be delivered to Rab5 and EEA1 positive early endosomes for further transport to late endosomes and lysosomes for degradation (Naslavsky et al. 2003). Recently, rafts were reported to be recycled by an Arf6-dependent process (Balasubramanian et al. 2007). Interestingly, the Herpes Simplex Virus protein VP22 was in CHO-K1 and HeLa cells shown to be endocytosed by a mechanism dependent on Arf6, dynamin and lipid rafts, but independent of Rho-GTPases (Nishi and Saigo 2007).

A new class of trafficking motifs in non-clathrin-dependent cargo proteins have been identified and suggested to prevent internalization and/or facilitate recycling of pro- teins carrying these, via the Arf6-regulated pathway by enhancing their association with the Arf6-specific guanine nucleotide exchange factor EFA6 (Gong et al. 2007). In certain cell types, rather than defining a separate endocytic pathway, Arf6 regulates clathrin-dependent uptake. In Hek293 cells, for example, Arf6 regulates uptake of several G-protein coupled receptors by clathrin-dependent endocytosis (Houndolo et al. 2005), and there is also evidence for complex formation between Arf6, the $\beta$-subunit of AP2 and the heavy chain of clathrin (Poupart et al. 2007). The data furthermore suggest that Arf6 regulates the angiotensin AA type 1 receptor by recruiting AP2 and clathrin. Arf6 has also in HeLa cells been proposed to have a direct function in clathrin coat recruitment to the plasma membrane. In fact, Arf6-GTP has recently been demonstrated to interact with AP-2 both in vitro and in vivo, and a clathrin-interacting Arf6-specific GTPase activating protein (called SMAP), affecting clathrin-dependent endocytosis only, has also been identified (Paleotti et al. 2005; Tanabe et al. 2005). Interestingly, in polarized MDCK cells, Arf6 associated with the guanine nucleotide exchange factor ARNO regulates endocytosis exclusively at the apical surface by recruiting actin to clathrin-coated pits (Hyman et al. 2006).

The present picture is complicated as many of the roles ascribed to Arf6 within the endocytic system seem to be not only cell type dependent, but also determined by the exchange factor associated with Arf6 (Kalia et al. 2006). The Arf6 GTP exchange factor ARNO has previously been shown to be dependent on inositol-lipids for its membrane recruitment. However, the process seems to be even more complex as small $\mathrm{G}$ proteins of the Arl 4 family can function in recruitment of the Arf6 GEF cytohesin to the plasma membrane (Hofmann et al. 2007). Perhaps this might explain some of the apparent differences between cell types.

\section{Caveolae and endocytosis}

Caveolae are characteristic, small $(50-100 \mathrm{~nm})$, regular invaginations of the plasma membrane as seen in the EM (Fig. 3). Since caveolae have this distinct, easily recognizable ultrastructural morphology, they have been of particular interest in relation to clathrin-independent endocytosis. The distinct shape of caveolae early led to the assumption that caveolae were endocytic structures comparable to clathrin-coated pits and vesicles. Also, in thin sections for EM which are not cut perpendicular to the plasma membrane, one might get the impression of several free, i.e. not surface-connected, caveolar profiles, supporting the idea of caveolar endocytosis and a front of internalized caveolae moving into the cell (Fig. 3). Interestingly, more careful EM examination-for instance using Ruthenium Red as a surface marker-reveal that most if not all such vesicular 
Fig. 3 Appearance of caveolae in myoepithelial cells. In (a) is seen a single caveola (Cav) and for comparison a clathrin-coated pit $(C p)$. b shows a group of caveolae at the plasma membrane; as the section is largely perpendicular to the membrane, most of the caveolae are clearly seen to be surface-connected. In (c) is shown an example of a section which is not perpendicular to the plasma membrane, and here many of the caveolae appears as free (not surface-connected) vesicles. If such "free" vesicles should be unequivocally established as caveolae, they can be immunogold-labeled using an antibody against caveolin, as shown on the ultracryosection in (d) (Cav). An unlabeled, Cp is also seen. Bars $500 \mathrm{~nm}$
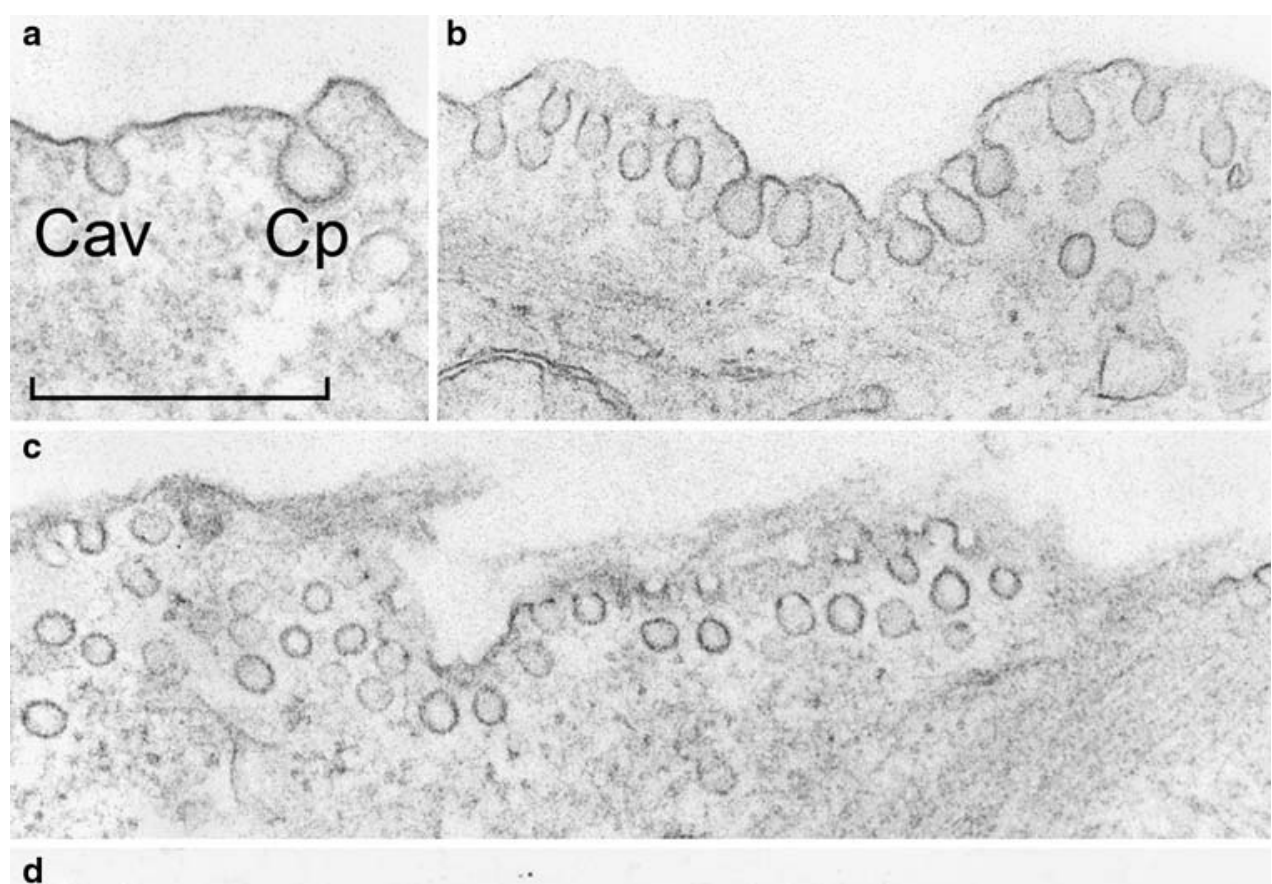

d

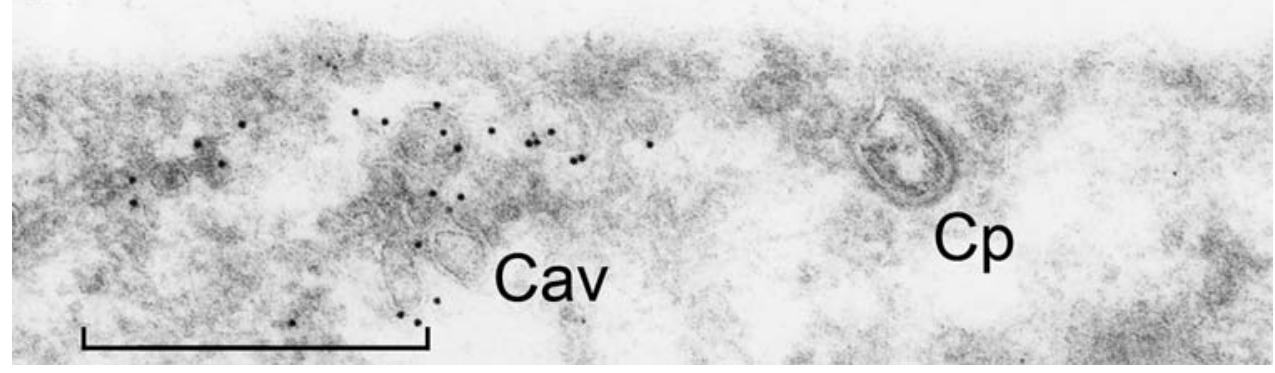

profiles are indeed surface-connected caveolae (Fig. 4). Also, the observation that e.g. Cholera toxin localized to caveolae supported the idea that caveolae were responsible for the endocytic uptake of Cholera toxin, a notion that is certainly an oversimplification (Torgersen et al. 2001; Shogomori and Futerman 2001; Nichols et al. 2001; see discussion in Hommelgaard et al. 2005; see also Kirkham et al. 2005).

However, fluorescence recovery after photobleaching (FRAP) analysis of cells expressing GFP-caveolin revealed that caveolae under normal conditions are not involved in endocytosis to any significant degree (Thomsen et al. 2002). Similarly, more recently live cell imaging of cells expressing GFP-caveolin showed that even high concentrations of EGF, which had been reported to lead to internalization of the EGFR via caveolae (Sigismund et al. 2005), do not mobilize caveolae above a low background level (Kazazic et al. 2006). Also, in a careful EM study where the setup allowed to discriminate between caveolin-associated and other vesicles as well as between surface-connected and free vesicles, Kirkham et al. (2005) showed that only about $2 \%$ of caveolae bud from the plasma membrane per minute. For comparison, it takes $50-150 \mathrm{~s}$ for a clathrin- coated pit to assemble and pinch off to form a free, clathrincoated vesicle (Ungewickell and Hinrichsen 2007). This means that the entire population of coated pits present at a given time point will have been internalized within approximately 1-2.5 min. Thus, today it appears well-established and generally accepted that under normal, non-stimulating conditions, caveolae are quite stable structures at the plasma membrane that are not involved in endocytosis to any significant degree (van Deurs et al. 2003; Pelkmans et al. 2004; Hommelgaard et al. 2005; Parton et al. 2006; Kirkham et al. 2005; Lajoie and Nabi 2007).

On the other hand, it is also evident from the above mentioned studies that a few caveolae may pinch off from the plasma membrane (Thomsen et al. 2002; Kirkham et al. 2005). This could simply be due to a basal turnover of caveolae at the plasma membrane. Normally, caveolae seem to be stabilized at, or anchored to, the plasma membrane by actin filaments (Stahlhut and van Deurs 2000; Hommelgaard et al. 2005; Thomsen et al. 2002; Pelkmans et al. 2002). The phosphatase inhibitor okadaic acid stimulates caveolar clustering and internalization in an actin-dependent manner (Parton et al. 1994; Thomsen et al. 2002). One could therefore also imagine that caveolae might be 
Fig. 4 Caveolae are surfaceconnected structures. a-d show filter-grown MDCK cells postfixed with the electron-dense cell surface marker Ruthenium Red from the basolateral side. In (a) is seen a number of caveolae connected to the basolateral membrane (arrowheads). Also note that three caveolae (arrows) are connected to the plasma membrane (open arrow) via a larger membrane invagination (asterisk). b-d show clusters of caveolae (arrows) associated with elongated "vacuolar" structures (asterisks) apparently freely localized in the cytoplasm. However, the labelling with Ruthenium Red clearly demonstrates that these structure are indeed connected to the basolateral membrane, as revealed in (a). En, early and later endosomes; $\mathrm{Nu}$, nucleus. Bar $500 \mathrm{~nm}$
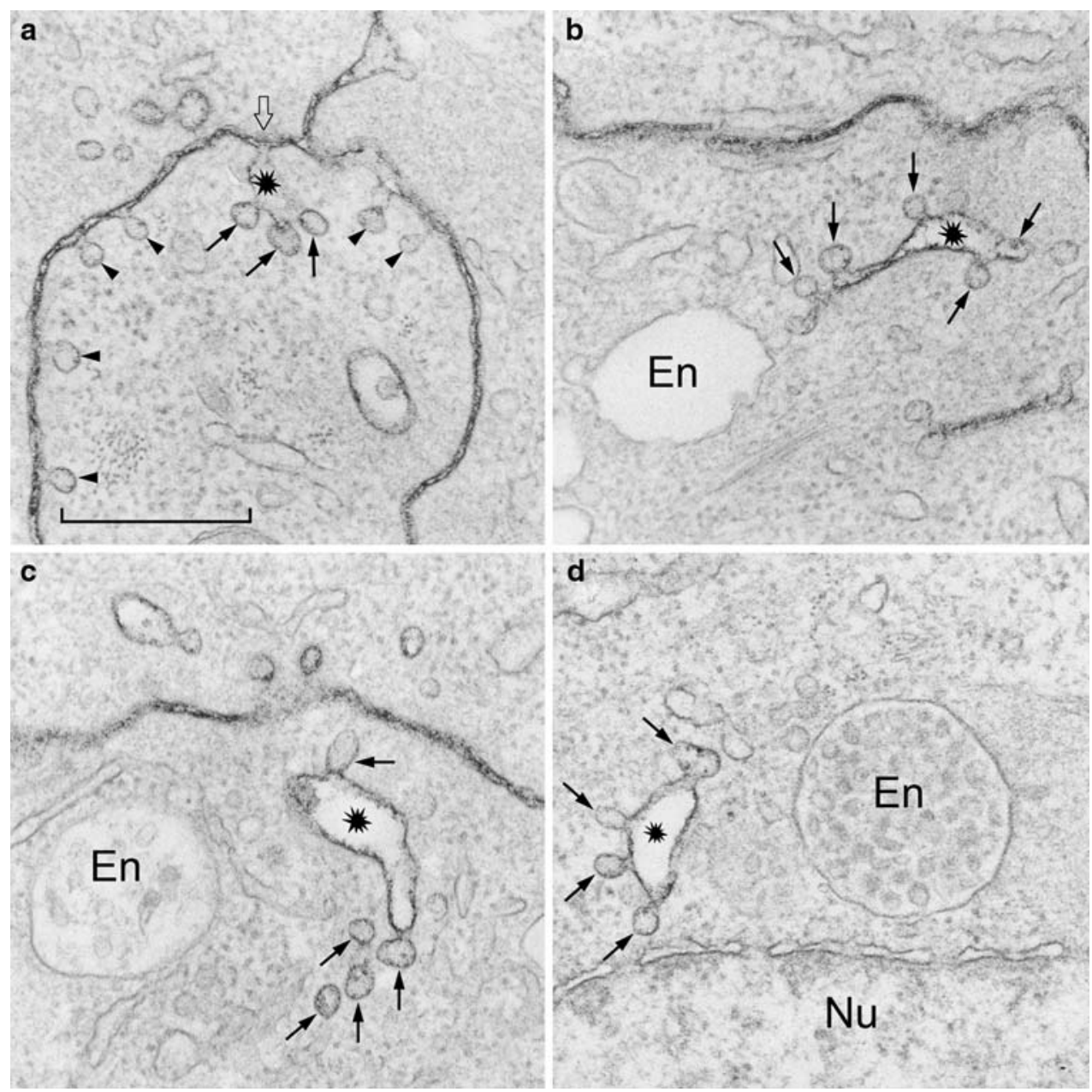

involved in a very slow, regulated endocytic uptake which under certain circumstances can be speeded up, for instance by controlled reorganization of the actin cytoskeleton. However, this remains speculative.

Interestingly, it was recently reported that coassembly of flotillin- 1 and -2 induced membrane curvature and formation of membrane invaginations morphologically similar to caveolae but different from these by not containing caveolin, the major caveolar protein. These flotillin-positive but caveolin-negative structures became internalized in contrast to "classical" caveolae (Frick et al. 2007).

Caveolae perform short range "kiss-and-run" motility very close to the plasma membrane. The process most likely involves continuous internalization and recycling of caveolae immediately beneath the plasma membrane (probably in the narrow 100-200 $\mathrm{nm}$ zone between the plasma membrane and the stabilizing actin cytoskeleton) (Thomsen et al. 2002, Fig. 5, online movie; Pelkmans and Zerial 2005). The significance of this process is unclear but it may explain the widely accepted role of caveolae in endothelial transcytosis. Thus, since endothelial cells are often very flat (100-200 nm), caveolae that have pinched off from e.g. the luminal endothelial plasma membrane, could fuse immediately after with the ablumenal plasma membrane or, in cases where the endothelial cell is very flat, the fusion may take place even before the pinching has been completed, and temporary transendothelial channels may arise (van Deurs et al. 2003).

When SV40 virus is added to cells, the virus particles are trapped in caveolae which subsequently become internalized by a process which requires tyrosine phosphorylation, reorganization of the cortical actin cytoskeleton, and recruitment of dynamin (Pelkmans et al. 2001, 2002). Characteristically, the caveolar internalization is a single wave phenomenon and it takes hours before caveolin/caveolae reappear at the plasma membrane. More recently, it was shown that SV40 virus is actually internalized much more efficiently by a caveolin-independent mechanism (Damm et al. 2005). In any case it is questionable whether the downregulation of plasma membrane caveolae induced by SV40 virus represents any normally occurring, inducible clathrin-independent endocytic mechanism. Rather, it might reflect clearance of the plasma membrane for caveolae occupied by cross-linked or aggregated receptors or 
ligands. Such downregulation of caveolae stimulated by receptor cross-linking is discussed in more detail elsewhere (see Hommelgaard et al. 2005).

In this context, it is important to consider the many nonendocytic functions that have been associated with plasma membrane caveolae (see van Deurs et al. 2003). Thus, endothelial nitric oxide synthase (eNOS) is associated with caveolin-1, and caveolae play an important role in NO signaling, and are also involved in e.g. calcium signalling (Murata et al. 2007). Moreover, caveolae is important for cholesterol transport and homeostasis in adipocytes, and it was recently found that cholesterol-induced transport of caveolin to lipid droplets requires dynamin and PKC and very likely involves caveolar endocytosis (Le et al. 2006). Therefore, rather than considering caveolae in general as structures specialized for clathrin-independent endocytosis, they should be considered multifunctional membrane domains with physiological roles depending on cell type and situation (van Deurs et al. 2003). In addition, a recent report suggests that caveolins may even regulate cell function independently of caveolae (Head and Insel 2007).

\section{Regulation of endocytosis in polarized epithelial cells}

Studies of endocytic mechanisms in cell lines do not necessarily reflect the uptake mechanisms operating in vivo. Both cell density, cell adhesion and polarization can be regulators of endocytosis (del Pozo and Schwartz 2007; Sandvig 1978; Sandvig and van Deurs 2005; Eker et al. 1994; Holm et al. 1995; Llorente et al. 2000; Sandvig et al. 2000; Llorente et al. 1996). An early argument against clathrinindependent endocytosis was, not unexpectedly, that this could be a process occurring only in unpolarized cell lines grown on plastic support. However, investigations of polarized cells, such as MDCK I cells grown on filters, revealed that clathrin-independent endocytosis contributed to the endocytic uptake both on the apical and basolateral side (Eker et al. 1994). When using cytosolic acidification to block clathrin-dependent endocytosis (Sandvig et al. 1987), there was still uptake of the plant toxin ricin at both poles (Eker et al. 1994). The fact that there was a larger reduction of uptake at the apical side than at the basolateral side, does, however, not necessarily provide information about the fraction of clathrin-independent endocytosis at the two poles, since the low $\mathrm{pH}$ might affect the remaining uptake at the two poles differently. Importantly, apical clathrin-independent endocytosis turns out to be under complex regulation, and can be regulated independently of uptake at the basolateral side. Using either cells permeabilized at the basolaterial side or membrane permeable compounds (for review, see Sandvig and van Deurs 2005), we have shown that apical clathrin-independent endocytosis is regulated by protein kinase A (Eker et al. 1994), protein kinase C (Holm et al. 1995), phospholipase D (Sandvig et al. 2000), cyclooxygenase (Llorente et al. 2000) and calmodulin (Llorente et al. 1996). Interestingly, a Rho family member, apparently RhoA, seems to regulate apical endocytosis in a manner which can be supported by GTP $\gamma$ S (Garred et al. 2001). The RhoA-mediated mechanism is able to mediate uptake of both gold particles, ricin-HRP and HRP.

Acknowledgments Work performed by the authors of the present article is supported by the Norwegian and Danish Cancer Societies, the Norwegian Research Council for Science and the Humanities, The National Programme for Research in Functional Genomics in Norway (FUGE) in The Research Council of Norway, the Danish Medical Research Council, the Novo-Nordisk Foundation, and the John and Birthe Meyer Foundation.

\section{References}

Balasubramanian N, Scott DW, Castle JD, Casanova JE, Schwartz MA (2007) Arf6 and microtubules in adhesion-dependent trafficking of lipid rafts. Nat Cell Biol

Balklava Z, Pant S, Fares H, Grant BD (2007) Genome-wide analysis identifies a general requirement for polarity proteins in endocytic traffic. Nat Cell Biol 9:1066-1073

Benmerah A, Lamaze C (2007) Clathrin-coated pits: vive la difference? Traffic 8:970-982

Bonazzi M, Spano S, Turacchio G, Cericola C, Valente C, Colanzi A, Kweon HS, Hsu VW, Polishchuck EV, Polishchuck RS, Sallese M, Pulvirenti T, Corda D, Luini A (2005) CtBP3/BARS drives membrane fission in dynamin-independent transport pathways. Nat Cell Biol 7:570-580

Brown FD, Rozelle AL, Yin HL, Balla T, Donaldson JG (2001) Phosphatidylinositol 4,5-bisphosphate and Arf6-regulated membrane traffic. J Cell Biol 154:1007-1017

Chadda R, Howes MT, Plowman SJ, Hancock JF, Parton RG, Mayor S (2007) Cholesterol-sensitive cdc42 activation regulates actin polymerization for endocytosis via the GEEC pathway. Traffic 8:702-717

Cheng ZJ, Singh RD, Sharma DK, Holicky EL, Hanada K, Marks DL, Pagano RE (2006) Distinct mechanisms of clathrin-independent endocytosis have unique sphingolipid requirements. Mol Biol Cell 17:3197-3210

Damke H, Baba T, van der Bliek AM, Schmid SL (1995) Clathrinindependent pinocytosis is induced in cells overexpressing a temperature-sensitive mutant of dynamin. J Cell Biol 131:69-80

Damm EM, Pelkmans L, Kartenbeck J, Mezzacasa A, Kurzchalia T, Helenius A (2005) Clathrin- and caveolin-1-independent endocytosis: entry of simian virus 40 into cells devoid of caveolae. J Cell Biol 168:477-488

del Pozo MA, Schwartz MA (2007) Rac, membrane heterogeneity, caveolin and regulation of growth by integrins. Trends Cell Biol 17:246-250

Delaney KA, Murph MM, Brown LM, Radhakrishna H (2002) Transfer of M2 muscarinic acetylcholine receptors to clathrin-derived early endosomes following clathrin-independent endocytosis. J Biol Chem 277:33439-33446

Doxsey SJ, Brodsky FM, Blank GS, Helenius A (1987) Inhibition of endocytosis by anti-clathrin antibodies. Cell 50:453-463

Eker P, Holm PK, van Deurs B, Sandvig K (1994) Selective regulation of apical endocytosis in polarized MDCK cells by mastoparan and cAMP. J Biol Chem 269:18607-18615 
Frick M, Bright NA, Riento K, Bray A, Merrified C, Nichols BJ (2007) Coassembly of flotillins induces formation of membrane microdomains, membrane curvature, and vesicle budding. Curr Biol 17:1151-1156

Fujimoto LM, Roth R, Heuser JE, Schmid SL (2000) Actin assembly plays a variable, but not obligatory role in receptor-mediated endocytosis in mammalian cells. Traffic 1:161-171

Gao YS, Hubbert CC, Lu J, Lee YS, Lee JY, Yao TP (2007) Histone deacetylase 6 regulates growth factor-induced actin remodeling and endocytosis. Mol Cell Biol 27:8637-8647

Garred O, Rodal SK, van Deurs B, Sandvig K (2001) Reconstitution of clathrin-independent endocytosis at the apical domain of permeabilized MDCK II cells: requirement for a Rho-family GTPase. Traffic 2:26-36

Gong Q, Weide M, Huntsman C, Xu Z, Jan LY, Ma D (2007) Identification and characterization of a new class of trafficking motifs for controlling clathrin-independent internalization and recycling. J Biol Chem 282:13087-13097

Grimmer S, van Deurs B, Sandvig K (2002) Membrane ruffling and macropinocytosis require cholesterol. J Cell Sci 115:2953-2962

Hanzal-Bayer MF, Hancock JF (2007) Lipid rafts and membrane traffic. FEBS Lett 581:2098-2104

Head BP, Insel PA (2007) Do caveolins regulate cells by actions outside of caveolae? Trends Cell Biol 17:51-57

Hofmann I, Thompson A, Sanderson CM, Munro S (2007) The Arl4 family of small $\mathrm{G}$ proteins can recruit the cytohesin Arf6 exchange factors to the plasma membrane. Curr Biol 17:711-716

Holm PK, Eker P, Sandvig K, van Deurs B (1995) Phorbol myristate acetate selectively stimulates apical endocytosis via protein kinase C in polarized MDCK cells. Exp Cell Res 217:157-168

Hommelgaard AM, Roepstorff K, Vilhardt F, Torgersen ML, Sandvig K, van Deurs B (2005) Caveolae: stable membrane domains with a potential for internalization. Traffic 6:720-724

Houndolo T, Boulay PL, Claing A (2005) G protein-coupled receptor endocytosis in ADP-ribosylation factor 6-depleted cells. J Biol Chem 280:5598-5604

Hyman T, Shmuel M, Altschuler Y (2006) Actin is required for endocytosis at the apical surface of Madin-Darby canine kidney cells where ARF6 and clathrin regulate the actin cytoskeleton. Mol Biol Cell 17:427-437

Kaksonen M, Toret CP, Drubin DG (2005) A modular design for the clathrin- and actin-mediated endocytosis machinery. Cell 123:305-320

Kaksonen M, Toret CP, Drubin DG (2006) Harnessing actin dynamics for clathrin-mediated endocytosis. Nat Rev Mol Cell Biol 7:404414

Kalia M, Kumari S, Chadda R, Hill MM, Parton RG, Mayor S (2006) Arf6-independent GPI-anchored protein-enriched early endosomal compartments fuse with sorting endosomes via a Rab5/ phosphatidylinositol-3'-kinase-dependent machinery. Mol Biol Cell 17:3689-3704

Kazazic M, Roepstorff K, Johannessen LE, Pedersen NM, van Deurs B, Stang E, Madshus IH (2006) EGF-induced activation of the EGF receptor does not trigger mobilization of caveolae. Traffic 7:1518-1527

Kirkham M, Fujita A, Chadda R, Nixon SJ, Kurzchalia TV, Sharma DK, Pagano RE, Hancock JF, Mayor S, Parton RG (2005) Ultrastructural identification of uncoated caveolin-independent early endocytic vehicles. J Cell Biol 168:465-476

Lajoie P, Nabi IR (2007) Regulation of raft-dependent endocytosis. J Cell Mol Med 11:644-653

Lanzetti L (2007) Actin in membrane trafficking. Curr Opin Cell Biol 19:453-458

Larkin JM, Brown MS, Goldstein JL, Anderson RGW (1983) Depletion of intracellular potassium arrests coated pit formation and receptor-mediated endocytosis in fibroblasts. Cell 33:273-285
Lauvrak SU, Wälchli S, Slagsvold HH, Torgersen ML, Spilsberg B, Sandvig K (2006) Shiga toxin regulates its entry in a Syk-dependent manner. Mol Biol Cell 17:1096-1109

Le LS, Hajduch E, Lindsay MR, Le LX, Thiele C, Ferre P, Parton RG, Kurzchalia T, Simons K, Dugail I (2006) Cholesterol-induced caveolin targeting to lipid droplets in adipocytes: a role for caveolar endocytosis. Traffic 7:549-561

Llorente A, Garred Ø, Holm PK, Eker P, Jacobsen J, van Deurs B, Sandvig K (1996) Effect of calmodulin antagonists on endocytosis and intracellular transport of ricin in polarized MDCK cells. Exp Cell Res 227:298-308

Llorente A, van Deurs B, Garred Ø, Eker P, Sandvig K (2000) Apical endocytosis of ricin in MDCK cells is regulated by the cyclooxygenase pathway. J Cell Sci 113:1213-1221

Madshus IH, T:nnessen TI, Olsnes S, Sandvig K (1987) Effect of potassium depletion of Hep 2 cells on intracellular $\mathrm{pH}$ and on chloride uptake by anion antiport. J Cell Physiol 131:6-13

Marsh M, Helenius A (2006) Virus entry: open sesame. Cell 124:729_ 740

Mayor S, Pagano RE (2007) Pathways of clathrin-independent endocytosis. Nat Rev Mol Cell Biol 8:603-612

McNiven MA (2006) Big gulps: specialized membrane domains for rapid receptor-mediated endocytosis. Trends Cell Biol $16: 487-492$

McPherson PS (2002) The endocytic machinery at an interface with the actin cytoskeleton: a dynamic, hip intersection. Trends Cell Biol 12:312-315

Montesano R, Roth J, Robert A, Orci L (1982) Non-coated membrane invaginations are involved in binding internalization of cholera and tetanus toxins. Nature 296:651-653

Moya M, Dautry-Varsat A, Goud B, Louvard D, Boquet P (1985) Inhibition of coated pit formation in Hep2 cells blocks the cytotoxicity of diphtheria toxin but not that of ricin. J Cell Biol 101:548-559

Murata T, Lin MI, Stan RV, Bauer PM, Yu J, Sessa WC (2007) Genetic evidence supporting caveolae microdomain regulation of calcium entry in endothelial cells. J Biol Chem 282:16631-16643

Naslavsky N, Weigert R, Donaldson JG (2003) Convergence of nonclathrin- and clathrin-derived endosomes involves Arf6 inactivation and changes in phosphoinositides. Mol Biol Cell 14:417-431

Naslavsky N, Weigert R, Donaldson JG (2004) Characterization of a nonclathrin endocytic pathway: membrane cargo and lipid requirements. Mol Biol Cell 15:3542-3552

Nichols BJ, Kenworthy AK, Polishchuk RS, Lodge R, Roberts TH, Hirschberg K, Phair RD, Lippincott-Schwartz J (2001) Rapid cycling of lipid raft markers between the cell surface and golgi complex. J Cell Biol 153:529-542

Nishi K, Saigo K (2007) Cellular internalization of green fluorescent protein fused with herpes simplex virus protein VP22 via a lipid raft-mediated endocytic pathway independent of caveolae and Rho family GTPases but dependent on dynamin and Arf6. J Biol Chem 282:27503-27517

Orth JD, McNiven MA (2006) Get off my back! Rapid receptor internalization through circular dorsal ruffles. Cancer Res 66:1109411096

Orth JD, Krueger EW, Weller SG, McNiven MA (2006) A novel endocytic mechanism of epidermal growth factor receptor sequestration and internalization. Cancer Res 66:3603-3610

Paleotti O, Macia E, Luton F, Klein S, Partisani M, Chardin P, Kirchhausen T, Franco M (2005) The small G-protein Arf6GTP recruits the AP-2 adaptor complex to membranes. J Biol Chem 280:21661-21666

Parton RG, Joggerst B, Simons K (1994) Regulated internalization of caveolae. J Cell Biol 127:1199-1215

Parton RG, Hanzal-Bayer M, Hancock JF (2006) Biogenesis of caveolae: a structural model for caveolin-induced domain formation. J Cell Sci 119:787-796 
Payne GS, Baker D, van Tuinen E, Schekman R (1988) Protein transport to the vacuole and receptor-mediated endocytosis by clathrin heavy chain-deficient yeast. J Cell Biol 106:1453-1461

Payne CK, Jones SA, Chen C, Zhuang X (2007) Internalization and trafficking of cell surface proteoglycans and proteoglycan-binding ligands. Traffic 8:389-401

Pelkmans L, Zerial M (2005) Kinase-regulated quantal assemblies and kiss-and-run recycling of caveolae. Nature 436:128-133

Pelkmans L, Kartenbeck J, Helenius A (2001) Caveolar endocytosis of simian virus 40 reveals a new two-step vesicular-transport pathway to the ER. Nat Cell Biol 3:473-483

Pelkmans L, Puntener D, Helenius A (2002) Local actin polymerization and dynamin recruitment in SV40-induced internalization of caveolae. Science 296:535-539

Pelkmans L, Burli T, Zerial M, Helenius A (2004) Caveolin-stabilized membrane domains as multifunctional transport and sorting devices in endocytic membrane traffic. Cell 118:767-780

Poupart ME, Fessart D, Cotton M, Laporte SA, Claing A (2007) ARF6 regulates angiotensin II type 1 receptor endocytosis by controlling the recruitment of AP-2 and clathrin. Cell Signal 19:2370-2378

Radhakrishna H, Donaldson JG (1997) ADP-ribosylation factor 6 regulates a novel plasma membrane recycling pathway. J Cell Biol 139:49-61

Rajendran L, Simons K (2005) Lipid rafts and membrane dynamics. J Cell Sci 118:1099-1102

Ridley AJ, Paterson HF, Johnston CL, Diekmann D, Hall A (1992) The small GTP-binding protein rac regulates growth factor- induced membrane ruffling. Cell 70:401-410

Rodal SK, Skretting G, Garred Ø, Vilhardt F, van Deurs B, Sandvig K (1999) Extraction of cholesterol with methyl-b-cyclodextrin perturbs formation of clathrin-coated endocytic vesicles. Mol Biol Cell 10:961-974

Römer W, Berland L, Chambon V, Gaus K, Windschiegl B, Tenza D, Aly MR, Fraisier V, Florent JC, Perrais D, Lamaze C, Raposo G, Steinem C, Sens P, Bassereau P, Johannes L (2007) Shiga toxin induces tubular membrane invaginations for its uptake into cells. Nature 450:670-675

Sandvig K (1978) Cell density affects the binding of the toxic lectin abrin to HeLa cells in monolayer cultures. FEBS Lett 89:233-236

Sandvig K (2001) Shiga toxins. Toxicon 39:1629-1635

Sandvig K, van Deurs B (1991) Endocytosis without clathrin (a minireview). Cell Biol Int Rep 15:3-8

Sandvig K, van Deurs B (1994) Endocytosis without clathrin. Trends Cell Biol 4:275-277

Sandvig K, van Deurs B (2002) Membrane traffic exploited by protein toxins. Ann Rev Cell Dev Biol 18:1-14

Sandvig K, van Deurs B (2005) Delivery into cells: Lessons learned from plant and bacterial toxins. Gene Ther 12:865-872

Sandvig K, Olsnes S, Petersen OW, van Deurs B (1987) Acidification of the cytosol inhibits endocytosis from coated pits. J Cell Biol 105:679-689

Sandvig K, Olsnes S, Petersen OW, van Deurs B (1988) Inhibition of endocytosis from coated pits by acidification of the cytosol. J Cell Biochem 36:73-81

Sandvig K, Llorente A, Rodal SK, Eker P, Garred O, Stahlhut M, van Deurs B (2000) Apical macropinocytosis in polarized MDCK cells: regulation by $N$-ethylmaleimide-sensitive proteins. Eur $\mathrm{J}$ Cell Biol 79:447-457
Sandvig K, Spilsberg B, Lauvrak SU, Torgersen ML, Iversen T-G, van Deurs B (2004) Pathways followed by protein toxins into cells. Int J Med Microbiol 293:483-490

Sandvig K, Sundan A, Olsnes S (1985) Effect of potassium depletion of cells on their sensitivity to diphtheria toxin and pseudomonas toxin. J Cell Physiol 124:54-60

Schmid EM, McMahon HT (2007) Integrating molecular and network biology to decode endocytosis. Nature 448:883-888

Shogomori H, Futerman AH (2001) Cholera toxin is found in detergent-insoluble rafts/domains at the cell surface of hippocampal neurons but is internalized via a raft-independent mechanism. J Biol Chem 276:9182-9188

Sigismund S, Woelk T, Puri C, Maspero E, Tacchetti C, Transidico P, Di Fiore PP, Polo S (2005) Clathrin-independent endocytosis of ubiquitinated cargos. Proc Natl Acad Sci USA 102:2760-2765

Skretting G, Torgersen ML, van Deurs B, Sandvig K (1999) Endocytic mechanisms responsible for uptake of GPI-linked diphtheria toxin receptor. J Cell Sci 112:3899-3909

Smythe E, Ayscough KR (2006) Actin regulation in endocytosis. J Cell Sci 119:4589-4598

Spilsberg B, Llorente A, Sandvig K (2007) Polyunsaturated fatty acids regulate Shiga toxin transport. Biochem Biophys Res Commun 364:283-288

Stahlhut M, van Deurs B (2000) Identification of filamin as a novel ligand for caveolin-1: evidence for the organization of caveolin1 -associated membrane domains by the actin cytoskeleton. Mol Biol Cell 11:325-337

Subtil A, Gaidarov I, Kobylarz K, Lampson MA, Keen JH, McGraw TE (1999) Acute cholesterol depletion inhibits clathrin-coated pit budding. Proc Natl Acad Sci USA 96:6775-6780

Tanabe K, Torii T, Natsume W, Braesch-Andersen S, Watanabe T, Satake M (2005) A novel GTPase-activating protein for ARF6 directly interacts with clathrin and regulates clathrin-dependent endocytosis. Mol Biol Cell 16:1617-1628

Thomsen P, Roepstorff K, Stahlhut M, van Deurs B (2002) Caveolae are highly immobile plasma membrane microdomains, which are not involved in constitutive endocytic trafficking. Mol Biol Cell 13:238-250

Torgersen ML, Skretting G, van Deurs B, Sandvig K (2001) Internalization of cholera toxin by different endocytic mechanisms. J Cell Sci 114:3737-3747

Ungewickell EJ, Hinrichsen L (2007) Endocytosis: clathrin-mediated membrane budding. Curr Opin Cell Biol 19:417-425

Utskarpen A, Slagsvold HH, Iversen T-G, Wälchli S, Sandvig K (2006) Retrograde transport of ricin is regulated by Rab6A/A' in a sequential manner. Traffic 7:663-672

van Deurs B, Roepstorff K, Hommelgaard AM, Sandvig K (2003) Caveolae: anchored, multifunctional platforms in the lipid ocean. Trends Cell Biol 13:92-100

Verkade P, Harder T, Lafont F, Simons K (2000) Induction of caveolae in the apical plasma membrane of Madin-Darby canine kidney cells. J Cell Biol 148:727-739

Vogel U, Sandvig K, van Deurs B (1998) Expression of caveolin-1 and polarized formation of cavolae in Caco-2 and MDCK II cells. J Cell Sci 111:825-832

Yarar D, Waterman-Storer CM, Schmid SL (2007) SNX9 couples actin assembly to phosphoinositide signals and is required for membrane remodeling during endocytosis. Dev Cell 13:43-56 